\title{
CIAPIN1 confers multidrug resistance through up-regulation of MDR-1 and Bcl-L in LoVo/Adr cells and is independent of p53
}

\author{
YA-FEI ZHANG ${ }^{1}$, XIAO-HUA LI ${ }^{2}$, YONG-QUAN SHI ${ }^{2}$, YU-YUN WU $^{1}$, NING LI $^{1}$, \\ QIANG HE $^{3}$, QING JI ${ }^{1}$, RONG-QUAN WANG ${ }^{1}$, SHI-MING YANG ${ }^{1}$ and DIAN-CHUN FANG ${ }^{1}$ \\ Department of ${ }^{1}$ Gastroenterology, and ${ }^{3}$ Hepatobiliary Surgery, Southwest Hospital, Third Military \\ Medical University, Chongqing 400038; ${ }^{2}$ State Key Laboratory of Cancer Biology and Xijing Hospital \\ of Digestive Diseases, Fourth Military Medical University, Xi'an 710032, P.R. China
}

Received November 19, 2010; Accepted December 30, 2010

DOI: $10.3892 / o r .2011 .1148$

\begin{abstract}
Recent investigations discovered that CIAPIN1 might be another drug resistance-associated molecule in cancer cells. However, the underlying mechanisms of CIAPIN1-related multidrug resistance (MDR) remain elusive. In the present study, we investigated the role and possible mechanisms of CIAPIN1 in MDR of human colon carcinoma LoVo/Adr cells which express the wild-type p53 gene. By using small interference RNA and gene transfection techniques, we found that knockdown of CIAPIN1 expression re-sensitized LoVo/Adr cells to anti-cancer drugs and up-regulation of CIAPIN1 in sensitive LoVo cells resulted in a distinct MDR phenotype. We further revealed that CIAPIN1 conferred the MDR phenotype in LoVo/Adr cells through up-regulating expression of MDR-1 (P-gp) and Bcl-xL. Finally, by analyzing the effect of inactivation of wild-type p53 on CIAPIN1-induced up-regulation of P-gp and Bcl-xL, we determined that CIAPIN1 could exhibit its MDR-related function independently of the p53 signaling pathway. Overall, the results presented here further suggest that over-expression of CIAPIN1 is an important mechanism of drug resistance in human cancers, even if not the sole one.
\end{abstract}

\section{Introduction}

Chemotherapy is the primary treatment for human cancer, but one clinically significant problem that often causes failure of chemotherapy is multidrug resistance (MDR). It is recognized that MDR arises from molecular changes, and a variety of mechanisms have been extensively studied in vivo and in vitro $(1,2)$. However, the complete mechanisms involved in MDR

Correspondence to: Dr Dian-Chun Fang, Department of Gastroenterology, Southwest Hospital, Third Military Medical University, Chongqing 400038, P.R. China

E-mail: fangdianchun@hotmail.com

Key words: CIAPIN1, multidrug resistance, LoVo/Adr, MDR-1, Bcl-xL, p53 have not been fully characterized, suggesting that there are still some unknown molecules and mechanisms responsible for MDR.

CIAPIN1 was initially identified as an apoptosis inhibitor with no homology to apoptosis regulatory molecules of the Bcl-2 family, caspase family or signal transduction molecules (3). Subsequently, Hao et al found that CIAPIN1 was related to the MDR of gastric cancer SGC7901/Adr cells, and supposed that mutant p53-dependent signal pathway might be involved in the underlying mechanisms of CIAPIN1-conferred MDR (4). However, recently, Li et al investigated the relationship between CIAPIN1 expression and the MDR in leukemia HL-60/Adr cells which has been determined to be a p53-null tumor cell line, and revealed that CIAPIN1 was related to the MDR phenotype as well (5). In this study, a wild-type p53 drug-resistant cell line LoVo/Adr was selected as the experimental model (6), and by this, we demonstrated that CIAPIN1 can also confer the MDR phenotype in LoVo/Adr cells through a mechanism involving up-regulation of MDR-1 (P-gp) and Bcl-xL. Further, we investigated the role of wildtype p53 in the CIAPIN1-conferred MDR, and determined that in LoVo/Adr cells CIAPIN1 exhibits its MDR-related function independent of the p53 signaling pathway.

\section{Materials and methods}

Cell culture. Human colon carcinoma cell line LoVo and the adriamycin-resistant counterpart LoVo/Adr (both maintained in our laboratory) were used in this study. Details of the establishment and biological properties have been described (7-9). The cells were routinely cultured in RPMI-1640 medium (Life Technologies, Gaithersburg, MD) supplemented with $10 \%$ FBS (Life Technologies, Gaithersburg, MD) at $37^{\circ} \mathrm{C}$ in a $5 \% \mathrm{CO}_{2}$ incubator.

PCR analysis. Total cell RNA was extracted using TRIzol reagent (Invitrogen, USA) according to the manufacturer's protocol. Reverse transcription was performed on $1 \mu \mathrm{g}$ of total RNA from each sample using RevertAid ${ }^{\mathrm{TM}}$ First Strand cDNA synthesis kit (Fermentas, Canada). CIAPIN1 cDNA was then amplified using the following primer pairs: forward, 5'-CGGAATTCATGGCAGATTTTGGGATCTC-3'; reverse, 
5'-GGTCGACCTAGGCATCAAGATTGCTATC-3'. B-actin was used as the loading control. All PCR products were separated on ethidium bromide stained agarose gels and visualized with UV.

Immunocytochemistry assay. Cells were cultured on glass cover slips for $24 \mathrm{~h}$ and fixed with $4 \%$ paraformaldehyde. The fixed cells were rinsed in PBS and permeabilized with $0.1 \%$ Triton X-100 in PBS for 5 min. Endogenous peroxidase activity was blocked by incubation with $3 \%$ hydrogen peroxide for $10 \mathrm{~min}$ and rinsed in PBS. The slips were incubated with anti-CIAPIN1 primary mouse monoclonal antibody (developed by Hao et al) overnight at $4^{\circ} \mathrm{C}(10)$. Histostain ${ }^{\mathrm{TM}}$-Plus kits (Zhongshan, China) were used according to the manufacturer's instructions for detection of CIAPIN1.

Western blot analysis. Total cell lysate was prepared in $1 \mathrm{X}$ SDS buffer. Proteins were separated by SDS-PAGE and transferred onto PDVF membranes. Membranes were then blotted with antibodies to MDR-1 (P-gp), Bcl-2, Bax, p53 (DO-1) (Santa Cruz Biotechnology, Santa Cruz, CA), Bcl-xL (PharMingen, San Diego, CA), CIAPIN1 (10) and B-actin (Sigma, St. Louis, MO). Antigen-antibody complexes were visualized with the enhanced chemiluminescence (Amersham Pharmacia Biotech, Piscataway, NJ).

Cell transfection. Cells were planted on 6-well plates and cultured in drug-free medium. At $90-95 \%$ confluency, cells were washed twice with PBS and grown in 2 ml RPIM-1640 without antibiotics. Using Lipofectamine ${ }^{\mathrm{TM}} 2000$ reagent (Invitrogen, Inc., Carlsbad, CA, USA), $2 \mu \mathrm{g}$ pcDNA3.1 (Invitrogen) which included the full-length cDNA for CIAPIN1, or pSip53 small interfering RNA (siRNA) plasmids was transfected into LoVo cells, respectively, and $2 \mu \mathrm{g}$ pSiCIAPIN1 siRNA plasmids were transfected into LoVo/Adr cells, as described previously $(4,11)$. The cells that had been transfected with pcDNA3.1 vector alone or pSilencer ${ }^{\mathrm{TM}} 3.1-\mathrm{H} 1 \mathrm{Neo}$ vector alone served as negative control. Forty-eight hours later, cells were placed in growth medium containing G418 (Life Technologies) at a final concentration of $400 \mu \mathrm{g} / \mathrm{ml}$ for clone selection. The expression levels of CIAPIN1 in G418-resistant stable clones were evaluated by Western blot analysis.

Clonogenic assay. Cells were plated out at $5 \times 10^{5}$ cells per $100-\mathrm{mm}$ dishes the day before treatment, and then treated with different concentrations of adriamycin (between 0 and $20 \mu \mathrm{g} / \mathrm{ml}$ ) for $\sim 4 \mathrm{~h}$ in growth medium, passaged 3 days later at very low density and further cultured for 10 days without medium renewal. At this time, cells were fixed and stained with a solution of crystal violet in methanol-acetic acid, and the numbers of clones per dish were counted. Results are given as the calculated total number of cell clones that would have resulted from the plating of all the treated cells or as the calculated cloning efficiency (12).

In vitro drug sensitivity assay. Drug sensitivity was evaluated using the 3-(4,5-dimethylthiazol-2-yl)-2, 5-diphenyltetrazolium bromide (MTT) assay, as described previously (13). Briefly, cells were trypsinized and diluted with culture medium to seeding density (10,000 cells per well), suspended in 96-well plates ( $200 \mu 1 /$ well, Costar, Cambridge, MA, USA), and incubated at $37^{\circ} \mathrm{C}$ for $24 \mathrm{~h}$. Cells were then incubated for $72 \mathrm{~h}$ in the presence or absence of various concentrations of the four freshly prepared anti-cancer drugs (adriamycin, vincristine, 5-fluorouracil and mitomycin C) in $200 \mu \mathrm{l}$ medium. Each condition was assayed in triplicate. Following cell incubation for $72 \mathrm{~h}, 50 \mu \mathrm{l}$ of $2 \mathrm{mg} / \mathrm{ml}$ MTT (Sigma) was added into each well, and cells incubated another $4 \mathrm{~h}$. The supernatants were then discarded, and $150 \mu 1$ DMSO (Sigma) was added into each well to dissolve crystals. Absorbance at $490 \mathrm{~nm}\left(\mathrm{~A}_{490}\right)$ was measured with a microplate reader (BP800; Biohit, Helsinki, Finland). Cell survival rates were calculated according to the following formula: survival rate $=\left(\right.$ mean $\mathrm{A}_{490}$ of treated wells $/$ mean $\mathrm{A}_{490}$ of untreated wells $)$ $\mathrm{x} 100 \%$. Dose-response curves of the anti-cancer drugs were drawn on semilogarithmic coordinate paper, and $\mathrm{IC}_{50}$ values were determined. Resistance index (RI) was calculated using the following formula: $\mathrm{RI}=\mathrm{IC}_{50}$ for CIAPIN1-related LoVo transfectants $/ \mathrm{IC}_{50}$ for control cells.

Intracellular drug accumulation assay. Fluorescence intensity of intracellular adriamycin was determined by flow cytometry as previously reported (14). Cells at log phase were trypsinized, resuspended and plated into 6-well plates at a cell density of $1 \times 10^{6} /$ well. After overnight culture at $37^{\circ} \mathrm{C}$, adriamycin was added to the medium at a final concentration of $5 \mu \mathrm{g} / \mathrm{ml}$. The cells were incubated for $1 \mathrm{~h}$, and then were harvested by trypsinization for the determination of adriamycin accumulation, or cultured with fresh, drugfree medium for another $30 \mathrm{~min}$ for the determination of adriamycin retention. The harvested cells were washed with ice-cold PBS, and intracellular adriamycin was detected by flow cytometry with an excitation wavelength of $488 \mathrm{~nm}$ and an emission wavelength of $575 \mathrm{~nm}$. The experiment was performed independently 3 times. Finally, the adriamycinreleasing index of the colon carcinoma cells was calculated using the following formula: releasing index $=$ (accumulation value-retention value)/accumulation value.

Reporter gene assay. LoVo cells were seeded in 6-well plates and grown in maintenance medium. The PGL3MDR-1 vector (promoter of MDR-1, -136 to +10 ) and the control vector were established previously (15). Briefly, cells were co-transfected with indicated amounts of pcDNA3.1/ CIAPIN1 plasmids $(0.3,0.6$ and $0.9 \mu \mathrm{g})$, PGL3-MDR-1 and pRL-TK vector using the Fugene transfection regent (Roche, Indianapolis, IN, USA). After $48 \mathrm{~h}$, luciferase reporter assays were performed according to the manufacturer's instruction (Promega, Madison, WI, USA). Cells were then processed for B-galactosidase staining with PanVera B-galactosidase staining kit, according to the manufacturer's protocols. A total of 300 cells per well was countered, and the percentage of blue cells were determined.

Annexin V staining. Cells were washed twice with cold PBS and resuspended in $100 \mu \mathrm{l}$ binding buffer at a concentration of $1 \times 10^{6}$ cells $/ \mathrm{ml}$. Annexin V-FITC (5 $\mu 1$, PharMingen) and 10 of $20 \mu \mathrm{g} / \mathrm{ml}$ propidum iodide (Sigma) were then added to the cells. After incubation at room temperature for $15 \mathrm{~min}, 400 \mu \mathrm{l}$ Annexin-binding buffer was added to each sample, and the 


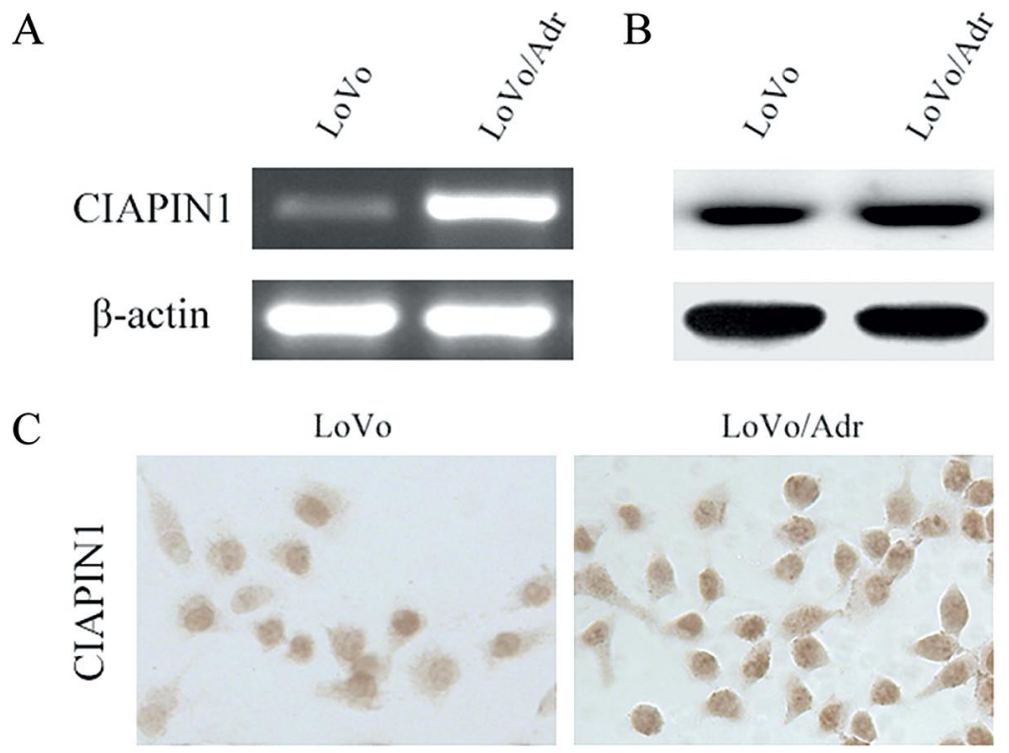

Figure 1. Expression of CIAPIN1 in LoVo/Adr and LoVo cells. Up-regulation of CIAPIN1 mRNA (A) and protein (B) was observed in LoVo/Adr cells compared with LoVo cells. B-actin was used as an internal control. Immunocytochemical staining (C) revealed stronger staining of CIAPIN1 in the nucleus and nucleolus of LoVo/Adr cells compared with that of LoVo cells (x200).

samples were kept on ice to counter the stained cells by flow cytometry (16).

Statistical analysis. The data are expressed as the means \pm SD. Differences were compared by one-way ANOVA analysis followed by LSD t-test. All statistical analyses were performed using SPSS11.0 software (Chicago, IL, USA). $\mathrm{P}<0.05$ was considered as statistically significant.

\section{Results}

The expression of CIAPIN1 is higher in LoVo/Adr cells than in LoVo cells at both the mRNA and protein level. RT-PCR and Western blot analysis revealed both mRNA and protein levels of CIAPIN1 were higher in LoVo/Adr than that in LoVo (Fig. 1A and B). CIAPIN1 was widely expressed both in cytoplasm and nucleus (17), and by immunocytochemistry (Fig. 1C), we found that although immunostaining for CIAPIN1 could be observed both in LoVo/Adr and LoVo cells, a much stronger staining was detected in the nucleus of LoVo/Adr cells. Of note, an intense accumulation of CIAPIN1 within nucleolus was observed in LoVo/Adr cells, whereas in LoVo cells, it was low or even absent.

Knockdown of CIAPIN1 expression resensitized LoVo/ Adr cells to anti-cancer drugs. To explore the relationship between over-expression of CIAPIN1 and the MDR phenotype, a siRNA eukaryotic expression vector of CIAPIN1 (pSiCIAPIN1) was transfected into LoVo/Adr cells (LoVo/ Adr-pSiCIAPIN1) to examine whether down-regulation of CIAPIN1 increased cell sensitivity towards anti-cancer drugs. As illustrated by Western blot analysis in Fig. 3E, CIAPIN1 protein level in LoVo/Adr-pSiCIAPIN1 cells reduced pronouncedly compared with that of parental LoVo/Adr cells. Clonogenic assay showed that LoVo/Adr-pSiCIAPIN1 cells displayed an obvious decrease in resistance to adriamycin
(Fig. 2A). Upon adriamycin exposure at $5 \mu \mathrm{g} / \mathrm{ml}$ for $4 \mathrm{~h}$, it appeared that the cloning efficiency of LoVo/Adr-pSiCIAPIN1 cells was on average 9.1-fold lower than that of the control cells transfected with pSilencer 3.1-H1 Neo vector (LoVo/Adr-pSi) $(\mathrm{P}<0.05)$ (Fig. 2B). Drug-sensitive assays in vitro revealed that down-regulation of CIAPIN1 increased the sensitivity of LoVo/Adr cells to three anti-cancer drugs with different mechanisms of action (P-gp-related drugs: adriamycin and vincristine, $\mathrm{P}<0.01$; $\mathrm{P}$-gp-unrelated drugs: mitomycin $\mathrm{C}$ and 5-fluorouracil, $\mathrm{P}<0.05, \mathrm{LoVo} / \mathrm{Adr}-\mathrm{pSiCIAPIN1}$ vs. LoVo/ Adr-pSi), which further suggested the MDR phenotype of LoVo/Adr cells could be partially reversed by knockdown of CIAPIN1 expression (Table I).

Up-regulation of CIAPIN1 in sensitive LoVo cells resulted in the MDR phenotype. Previous studies showed that transfection of CIAPIN1 was sufficient to confer adriamycin and vincristine resistance to leukemia and gastric cancer cells $(4,5)$. In the present study, drug-sensitive LoVo cells were transfected with the recombinant plasmids containing the complete open reading frame of CIAPIN1, and one stable clone with stable over-expression of CIAPIN1 (LoVopCIAPIN1) was selected (Fig. 3E). Fig. 2C showed the survival curve for adriamycin as analyzed by clonogenic assay. The rate of cell survival in LoVo-pCIAPIN1 (Fig. 2C and D), was significantly higher than that in LoVo-pc after exposure to $\geq 0.5 \mu \mathrm{g} / \mathrm{ml}$ adriamycin $(\mathrm{P}<0.05)$. Drug sensitivity assays in vitro showed that LoVo-pCIAPIN1 cells were 2.15-, 4.04-, and 8.58-fold more resistant relative to LoVo-pc cells to the three anti-cancer drugs adriamycin, vincristine and mitomycin $\mathrm{C}$, respectively, indicating that up-regulation of CIAPIN1 could induce an obvious MDR phenotype in drug sensitive LoVo cells (Table I).

CIAPIN1 up-regulated P-gp expression in LoVo cells and induced a corresponding MDR phenotype. Extrusion of 
A

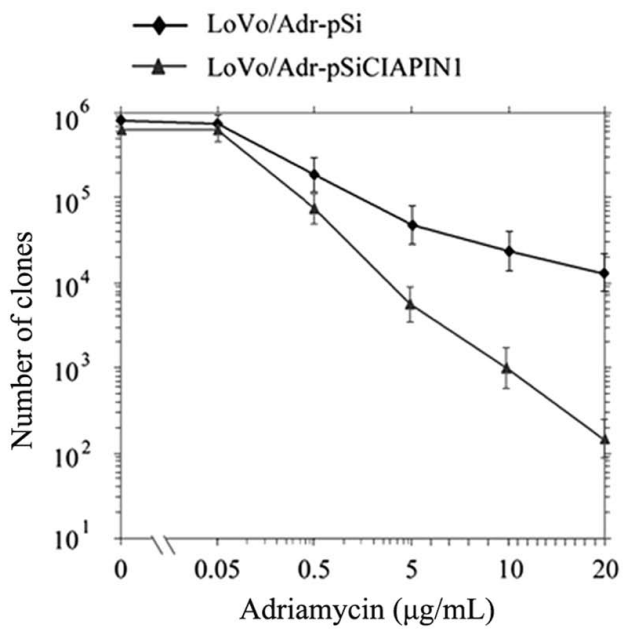

$\mathrm{C}$

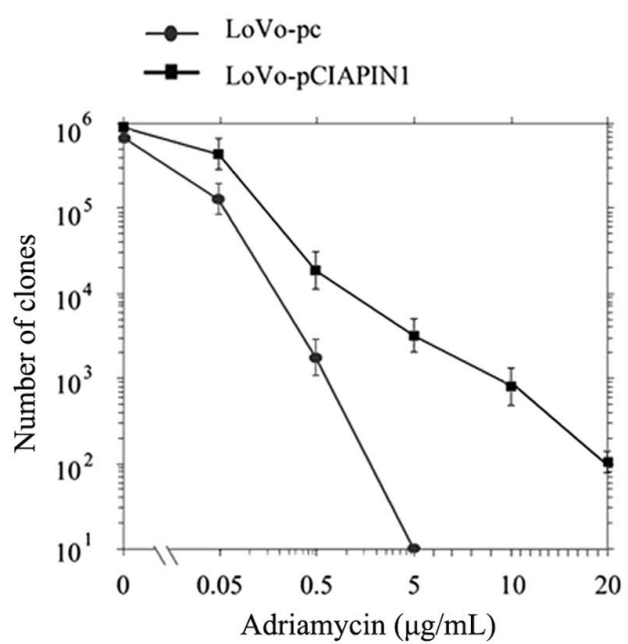

B

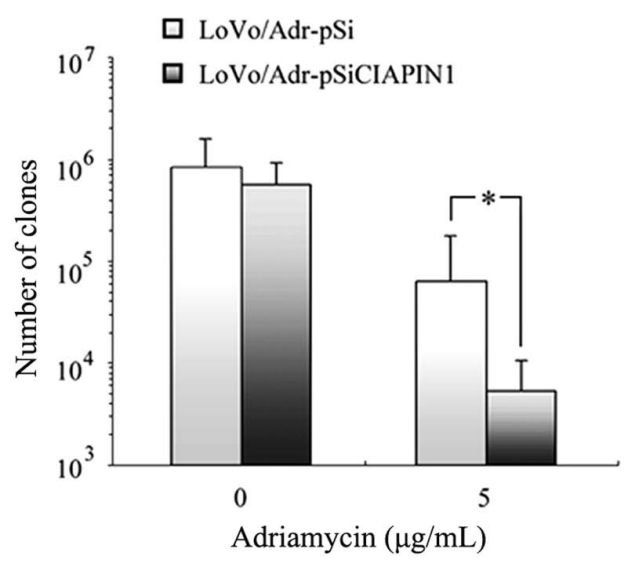

$\mathrm{D}$

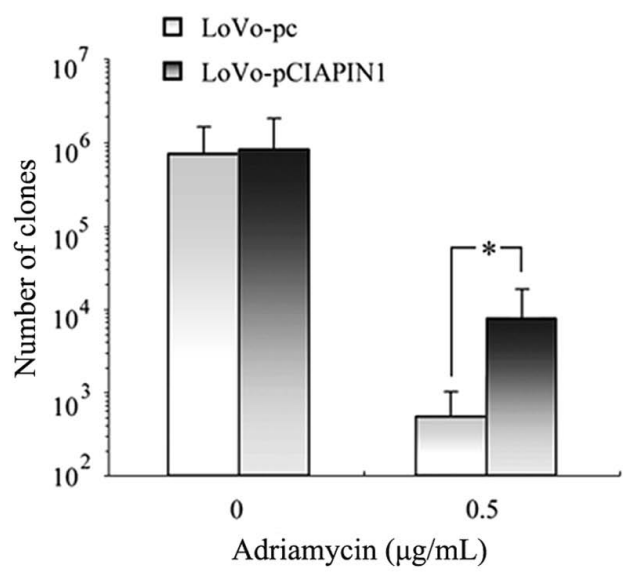

Figure 2. Results of clonogenic assay. Cells transfected with pSiCIAPIN1 or pCIAPIN1 were treated with the indicated adriamycin dose for $\sim 4$ h, and their cloning efficiency was measured as described in Materials and methods. Error bars indicate standard deviations measured with four independent cell populations. (A and B) Reversal of adriamycin resistance upon down-regulation of CIAPIN1 gene expression in LoVo/Adr cells. (C and D) Up-regulation of CIAPIN1 conferred adriamycin resistance to LoVo cells. ${ }^{*} \mathrm{P}<0.05$.

Table I. $\mathrm{IC}_{50}$ values $(\mu \mathrm{g} / \mathrm{ml})$ of anti-cancer drugs for colon carcinoma cells.

\begin{tabular}{|c|c|c|c|c|c|c|}
\hline \multirow[b]{3}{*}{ Drugs } & \multicolumn{6}{|c|}{ Cell lines } \\
\hline & \multicolumn{3}{|c|}{ LoVo/Adr } & \multicolumn{3}{|c|}{ LoVo } \\
\hline & $-p S i$ & -pSiCIAPIN1 & $\mathrm{RI}^{\mathrm{a}}$ & $-\mathrm{pc}$ & -pCIAPIN1 & $\mathrm{RI}^{\mathrm{a}}$ \\
\hline Adriamycin & $21.32 \pm 4.71$ & $4.92 \pm 1.35^{\mathrm{b}}$ & 0.23 & $0.41 \pm 0.09$ & $3.52 \pm 0.78^{\mathrm{b}}$ & 8.58 \\
\hline Vincristine & $183.03 \pm 34.23$ & $47.82 \pm 8.65^{\mathrm{b}}$ & 0.26 & $5.16 \pm 1.39$ & $32.79 \pm 6.71^{\mathrm{b}}$ & 6.35 \\
\hline Mitomycin C & $4.33 \pm 0.94$ & $2.77 \pm 0.51^{\mathrm{c}}$ & 0.64 & $0.59 \pm 0.11$ & $1.27 \pm 0.33^{\mathrm{c}}$ & 2.15 \\
\hline 5-fluorouracil & $0.62 \pm 0.11$ & $0.30 \pm 0.06^{c}$ & 0.49 & $0.55 \pm 0.10$ & $0.66 \pm 0.15$ & 1.20 \\
\hline
\end{tabular}

Survival rates of colon carcinoma cells to anti-cancer drugs were evaluated by MTT assay as described in Materials and methods. The doseeffect curves of anti-cancer drugs were drawn on semi-logarithm coordinate paper; thus, $\mathrm{IC}_{50} \mathrm{~S}$ were determined. Data are the mean $\pm \mathrm{SD}$ of four independent experiments. ${ }^{\mathrm{a} I}$ (resistance index) $=\mathrm{IC}_{50}$ for CIAPIN1-related LoVo transfectants $/ \mathrm{IC}_{50}$ for control cells. ${ }^{\mathrm{b}} \mathrm{P}<0.01$ and ${ }^{\mathrm{c}} \mathrm{P}<0.05$, CIAPIN1-related transfectants vs. control.

anti-cancer drugs by drug transporter P-gp is an important mechanism that confers the MDR phenotype in human cancers $(1,2,18)$, and previous studies have discovered that, compared with its parental LoVo cells, the most distinguished character of LoVo/Adr cells is the up-regulated expression of P-gp (7-9). In this experiment, we first estimated the expression 
A
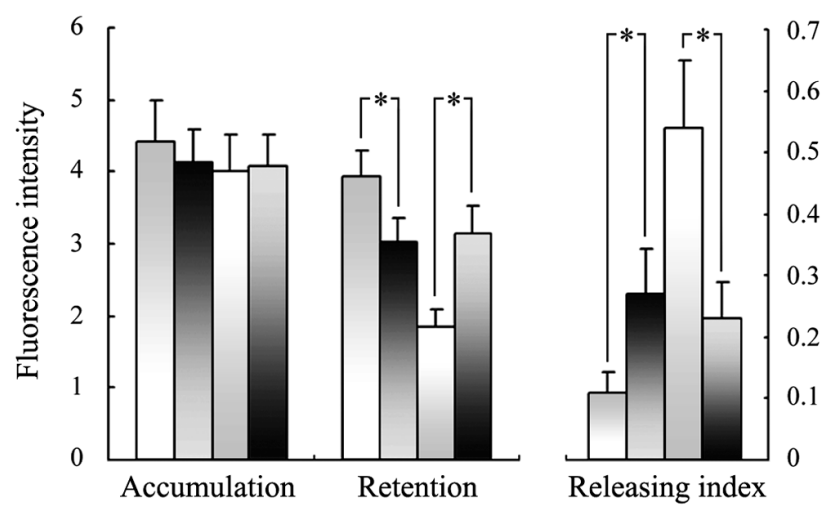

B

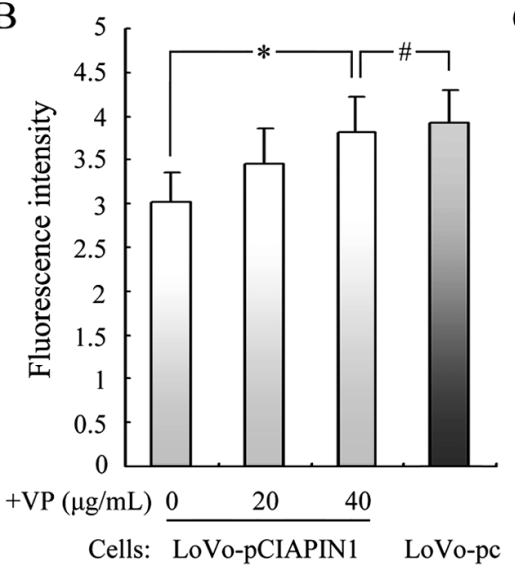

E

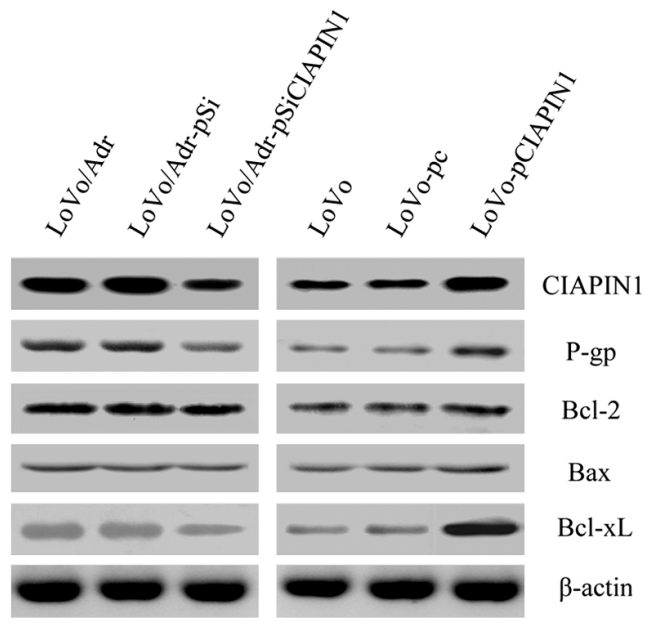

C

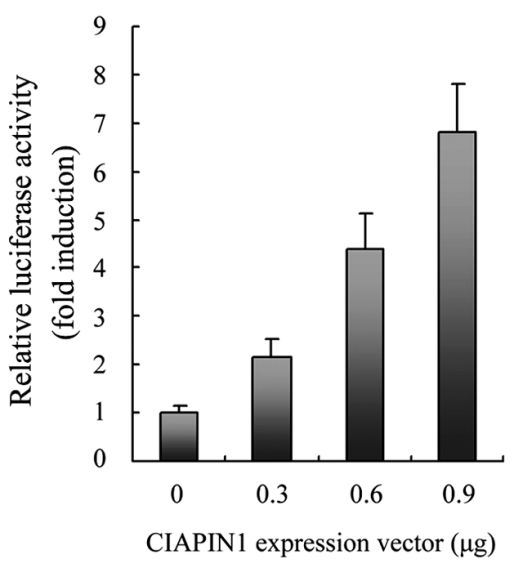

D

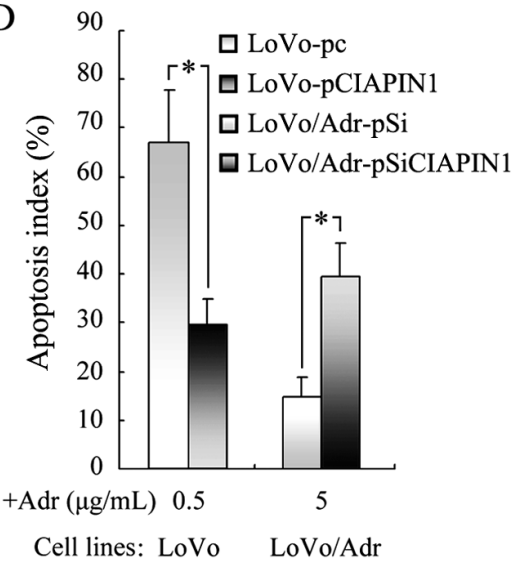

$\square$ LoVo-pc

口 LoVo-pCIAPIN1

$\square \mathrm{LoVo} / \mathrm{Adr}-\mathrm{pSi}$

口 LoVo/Adr-pSiCIAPIN1

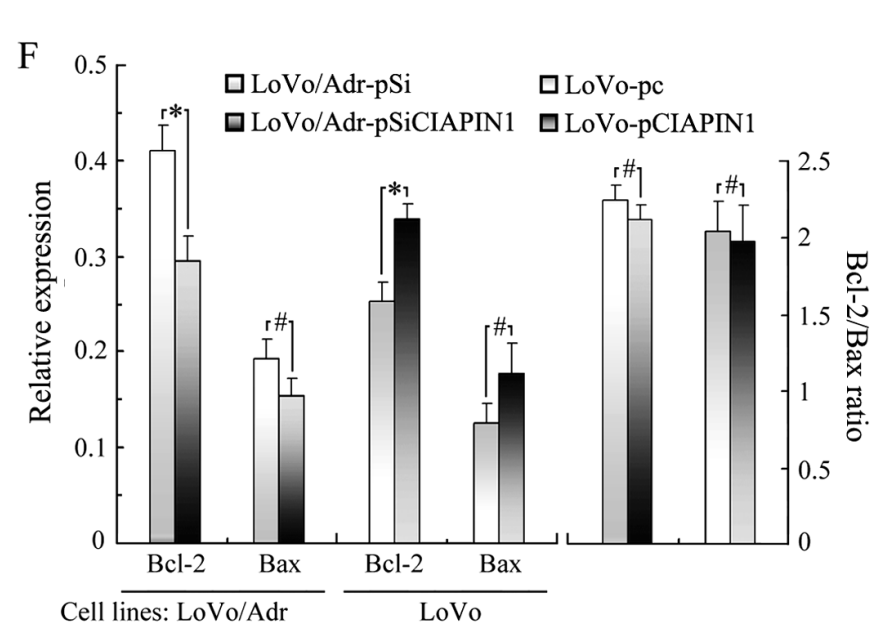

Figure 3. (A-C) P-gp over-expression is involved in CIAPIN1-induced multidrug resistance. (A) Fluorescence intensity of intracellular adriamycin was determined using flow cytometry as described in Materials and methods. Adriamycin-releasing index of the colon carcinoma cells was calculated using the following formula: releasing index $=$ (accumulation value-retention value)/accumulation value. $(\mathrm{B})$ The intracellular adriamycin retention of LoVo-pCIAPIN1 cells in the presence of verapamil (VP, $20 \mu \mathrm{g} / \mathrm{ml}$ or $40 \mu \mathrm{g} / \mathrm{ml}$ ) was analyzed for P-gp-related drug resistance. (C) Luciferase reporter assay was applied to determine the regulatory effect of CIAPIN1 on MDR-1 promoter activity. (D) Effect of CIAPIN1 on induction of apoptosis in response to adriamycin (Adr). Cells were collected $48 \mathrm{~h}$ after adriamycin treatment, and apoptosis was evaluated by flow cytometry. (A-D) Representative of four independent experiments. (E) Western blot analysis of CIAPIN1, P-gp, Bcl-2, Bax and Bcl-xL in colon carcinoma cells. B-actin was used as an internal control. Similar results were obtained in other two independent experiments. (F) Data of the relative expression of Bcl-2 and Bax were presented as expression relative to the level of the signal obtained with $\beta$-actin. Bcl-2/Bax ratio was determined by comparing the relative expression levels of Bcl-2 and Bax. Data represent the means \pm SD $(\mathrm{N}=3) .{ }^{*} \mathrm{P}<0.05 ;{ }^{*} \mathrm{P}>0.05$.

changes of P-gp in CIAPIN1-related LoVo transfectants. Western blot analysis revealed that the expression level of P-gp relative to $B$-actin was markedly higher in LoVopCIAPIN1 cells and lower in LoVo/Adr-pSiCIAPIN1 cells compared with the empty-vector-transfected control cells
(Fig. 3E). To test the relevance of this increase in P-gp expression to CIAPIN1-associated drug resistance in LoVo/Adr cells, we evaluated adriamycin accumulation and retention by flow cytometry in control and CIAPIN1-related transfectants. Adriamycin is fluorescent and this attribute provides easy 
A

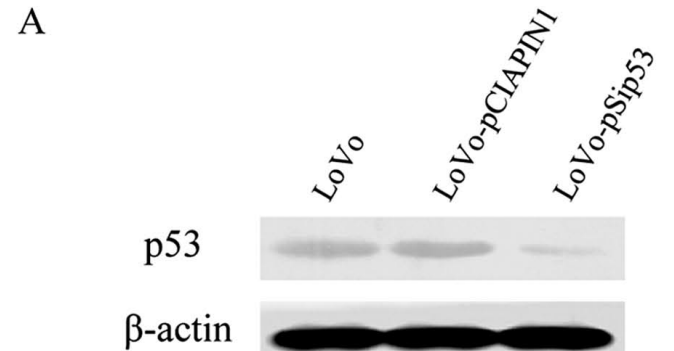

B

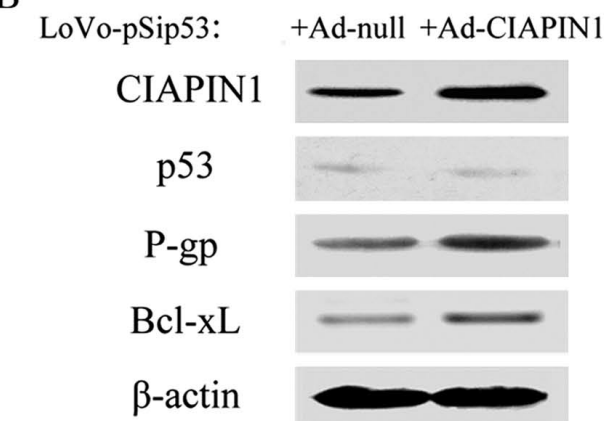

$\mathrm{C}$

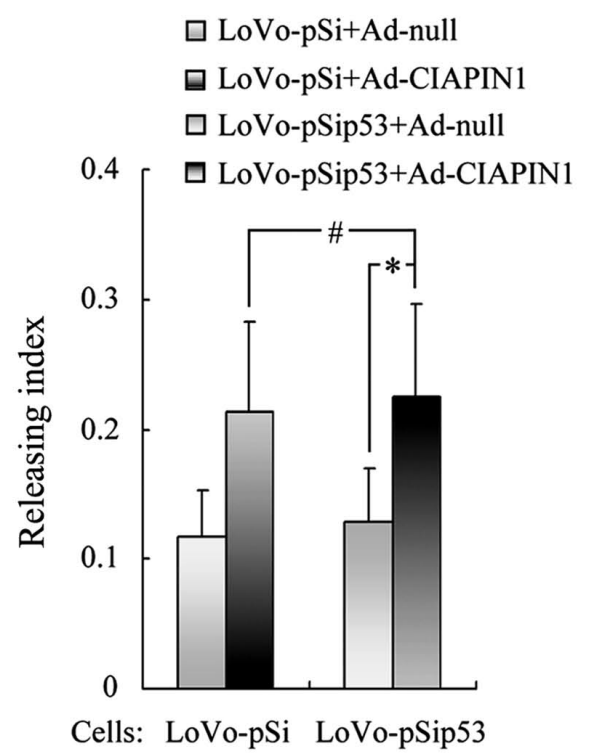

Figure 4. Wild-type p53 was not involved in CIAPIN1-mediated multidrug resistance in human colon carcinoma cells. (A) Expression level of p53 in LoVo-derived colon carcinoma cells. (B) Expression changes of P-gp and Bcl-xL in LoVo-pSip53 cells after exposure to Ad-CIAPIN1 at a dose of MOI 100 for $48 \mathrm{~h}$. Similar results were obtained in other two independent experiments. (C) After exposure to Ad-CIAPIN1 at a dose of MOI 100 for $48 \mathrm{~h}$, intracellular drug accumulation assay was applied to determine the adriamycin-releasing index in LoVo-pSip53 and its control LoVo-pSi cells. ${ }^{*} \mathrm{P}<0.05 ;{ }^{*} \mathrm{P}>0.05$.

monitoring of its intracellular accumulation and retention by flow cytometry. As shown in Fig. 3A, LoVo-pCIAPIN1 cells showed decreased adriamycin retention compared to LoVo-pc cells, while, the adriamycin retention in LoVo/ Adr-pSiCIAPIN1 cells was increased significantly compared to its control LoVo/Adr-pSi cells $(\mathrm{P}<0.05)$. Consistent with this, LoVo-pCIAPIN1 cells showed increased releasing index, whereas LoVo/Adr-pSiCIAPIN1 cells decreased. To confirm that the differences in drug accumulation were due to P-gp activity, we performed similar assay in LoVo-pCIAPIN1 cells in the presence of verapamil, an inhibitor of P-gp activity (15). As illustrated in Fig. 3B, verapamil could partially reduce the extrusion of adriamycin in LoVo-pCIAPIN1 cells.

Luciferase reporter assay was further performed in LoVo cells to investigate whether CIAPIN1 conferred the MDR through regulation of P-gp. As shown in Fig. 3C, co-transfection of the MDR-1 (P-gp) reporter gene with increasing amounts of the CIAPIN1 expression vector resulted in an essential liner increase in MDR-1 promoter activity, which suggested that CIAPIN1 was involved in regulation of MDR-1 transcription in LoVo/Adr cells.

CIAPINI increased the anti-apoptotic capacity of LoVo cells through up-regulation of $B c l-x L$. Suppression of drug-induced apoptosis is another important mechanism of MDR $(1,2,19)$. CIAPIN1 was initially discovered as a novel anti-apoptotic molecule (3), suggesting that up-regulation of CIAPIN1 might also contribute to the MDR phenotype through suppressing drug-induced apoptosis. In this study, to investigate whether CIAPIN1 affected the capacity of LoVo cells to undergo anti-cancer drugs induced apoptosis, Annexin V staining was performed. As revealed in Fig. 3D, the apoptosis index of LoVo-pCIAPIN1 cells was significantly lower than that of LoVo-pc cells after treatment of $0.5 \mu \mathrm{g} / \mathrm{ml}$ adriamycin for $48 \mathrm{~h}(\mathrm{P}<0.05)$, and on the other hand, LoVo/Adr-pSiCIAPIN1 cells showed increased apoptosis than that in its control LoVo/ Adr-pSi cells after treatment of $5 \mu \mathrm{g} / \mathrm{ml}$ adriamycin for $48 \mathrm{~h}$ $(\mathrm{P}<0.05)$.

For $\mathrm{Bcl}-2 / \mathrm{Bax}$ expression ratio is an important indicator of apoptosis (20), we tested the expression changes of the two molecules by Western blot analysis. As shown in Fig. 3E and $\mathrm{F}$, expression of the anti-apoptotic protein $\mathrm{Bcl}-2$ increased in response to up-regulation of CIAPIN1 $(\mathrm{P}<0.05)$. Interestingly, we observed that the pro-apoptotic protein Bax was also slightly up-regulated in LoVo-pCIAPIN1 cells, for which, there was no significant alternation of $\mathrm{Bcl}-2 / \mathrm{Bax}$ ratio observed in response to up-regulation of CIAPIN1 ( $P>0.05)$ (Fig. 3E and F). Because Shibayama et al observed that expression of Bcl-xL was severely impaired in the CIAPIN ${ }^{-/-}$mice (3), indicating that CIAPIN1 might regulate the expression of $\mathrm{Bcl}-\mathrm{xL}$, we further tested the expression of Bcl-xL and found that expression of Bcl-xL in pCIAPIN1-transfected LoVo cells was greatly up-regulated (Fig. 3E), suggested that CIAPIN1 increased the anti-apoptotic capacity of LoVo cells mainly through up-regulation of Bcl-xL.

CIAPIN1 conferred MDR in LoVo cells in a p53-independent manner. In a previous study, Li et al observed that, in p53 wild-type HepG2 and SMMC-7721 cells, apoptosis induced by AdSiCIAPIN1 (adenovirus-delivered CIAPIN1 small interfering RNA) partially resulted from accumulation of $\mathrm{p} 53$ protein, indicating CIAPIN1 might inhibit the expression or accumulation of p53 (11). For LoVo cells is known to express wild-type p53 (6), we investigated the role of p53 in the CIAPIN1-related MDR in present study. However, by Western blot analysis, we found that between LoVo-pCIAPIN1 and LoVo cells there was no obvious difference in $\mathrm{p} 53$ protein level (Fig. 4A). To elucidate whether CIAPIN1-conferred MDR in LoVo cells was p53-independent, we generated a LoVo cell line stably expressing antisense p53 (LoVo-pSip53) (Fig. 4A). 
As shown in Fig. 4B, inactivation of p53 in LoVo cells could not overcome the CIAPIN1-mediated expression changes of P-gp and Bcl-xL. Further, by intracellular drug accumulation assay, we revealed that, as well as that in LoVo-pSi cells, the adriamycin-releasing index in LoVo-pSip53 cells was significantly increased after exposure to Ad-CIAPIN1 (recombinant adenovirus expressing CIAPIN1, constructed in our laboratory) $(\mathrm{P}<0.05$, Fig. $4 \mathrm{C})(21)$, indicating that exogenous CIAPIN1 protein was of ability to up-regulate $\mathrm{P}-\mathrm{gp}$ expression and induce corresponding drug resistance phenotype without functional p53 protein.

\section{Discussion}

Increased amounts of P-gp may confer MDR on cells by preventing the intracellular accumulation of a variety of cytotoxic drugs, including adriamycin, vincristine and etoposide. The multidrug-resistant LoVo/Adr cells with wild-type p53 gene used in this study were selected by continuous exposure to adriamycin, whose classical phenotype was characterized by active drug extrusion due to P-gp over-expression (7-9). CIAPIN1 was found to be over-expressed in LoVo/Adr cells and confer the MDR phenotype. To clarify the association of P-gp with CIAPIN1-related MDR in LoVo/Adr cells, we investigated the effects of CIAPIN1 on the expression of $\mathrm{P}-\mathrm{gp}$ and the MDR phenotype mediated by P-gp. By using gene transfection and small interference RNA techniques, we demonstrated that CIAPIN1 could confer the MDR features of LoVo/Adr cells through up-regulating the expression of P-gp.

Suppression of drug-induced apoptosis is another important mechanism of MDR. By drug sensitivity assays, we revealed CIAPIN1 also conferred the MDR of LoVo/Adr cells to P-gp-unrelated drugs mitomycin $\mathrm{C}$, which indicated that over-expressed CIAPIN1 increased the anti-apoptotic capacity of LoVo/Adr cells as well. However, as analyzed by Western blot, both the expression of anti-apoptotic protein $\mathrm{Bcl}-2$ and apoptotic protein Bax increased in response to up-regulation of CIAPIN1, and from which, the Bcl-2/Bax ratio was not altered significantly. This result was not paralleled by the changes in MDR phenotype of LoVo-pCIAPIN1 cells. Therefore, we detected the expression of another anti-apoptotic protein Bcl-xL, and found it up-regulated in LoVo-pCIAPIN1 cells. Shibayama et al reported that the expression of Bcl-xL and JAK2 was severely impaired in the $\mathrm{CIAPIN}^{-/-}$mice, and hypothesized CIAPIN1 might protect cells against apoptosis through regulation of JAK2/STATs pathway (3). Although the significance of activation of the JAK2/STATs pathway remains under investigation, several reports have shown that phosphorylation and activation of JAK2 and STAT proteins appeared to play a critical role in regulating the expression of $\mathrm{Bcl}-2$ family proteins in human cancer cells, including the LoVo cells (22-24). Thus, the activation of JAK2/STATs pathway induced by CIAPIN1 seems to be a reasonable explanation for the increased expression of Bcl-xL, Bcl-2 and Bax in LoVo-pCIAPIN1 cells.

LoVo/Adr cells have been characterized as sensitive to 5-fluorouracil although displaying a cross-resistant phenotype to several anti-cancer drugs such as vincristine, VP-16, cyclophosphamide and mitomycin $C(9,25)$. In the present study, we also observed that up-regulation of CIAPIN1 in LoVo cells induced cross-resistance to vincristine and mitomycin $\mathrm{C}$, but not to 5-fluorouracil. In theory, up-regulation of CIAPIN1 and consequent increased Bcl-xL expression should increase the capacity of LoVo cells to undergo 5-fluorouracil-induced apoptosis. However, observations are inconsistent with the presumption. Interestingly, several multidrug-resistant cell lines, including gastric cancer SGC7901/Adr cells, leukemia HL-60/Adr cells, breast cancer MCF/Adr cells, and colon cancer LoVo/Adr cells, have been successfully selected by using similar methods that apply intermittent exposures to adriamycin. Among the four cell lines, SGC7901/Adr and leukemia HL-60 with p53 dysfunction for mutation or depletion showed cross-resistance to 5-fluorouracil $(26,27)$, whereas MCF/Adr and LoVo/Adr both with wild-type p53 was still sensitive to 5 -fluorouracil $(6,25,28)$. It has been discovered that 5-FU-induced apoptosis was accompanied by increased expression of Bax and Bak without consistent modulation of other Bcl-2 family proteins in a p53 (wild-type)-dependent manner (29). Therefore, we may infer that the anti-apoptotic effect of Bcl-xL in LoVo/Adr or LoVo-pCIAPIN1 cells is attenuated or eliminated by accompanied up-regulation of Bax and Bak in exposure to 5-fluorouracil.

Initially, Hao et al hypothesized that CIAPIN1 might confer MDR in cancer cells via regulating the expression of mutant p53 (4). However, it is worth noting that, the status of p53 in the three tested cancer cells was different: LoVo cells have p53 wild-type (6), SGC7901 cells are p53 mutant (27), and HL-60 cells are p53 null (28). Therefore, CIAPIN1 conferring MDR in cancer cells via regulating the expression of mutant p53 is not comprehensive. More recently, Li et al found that down-regulation of CIAPIN1 by adenovirusdelivered small interfering RNA could result in enhanced expression of p53 protein in HepG2 and SMMC-7721 cells that contained wild-type p53 gene (11). It seems reasonable that CIAPIN1 mediated MDR in wild-type p53 LoVo cells via inhibiting expression of p53 (30). However, by silencing p53 expression and analyzing the effect of inactivation of p53 on the CIAPIN1 induced up-regulation of P-gp and Bcl-xL, we excluded the possibility of CIAPIN1 conferring MDR via $\mathrm{p} 53$ signal pathway in LoVo/Adr cells. This result further verified the fact that CIAPIN1 could exhibit different functions in different genetic context in cell lines.

In conclusion, these results from our study further indicated that over-expression of CIAPIN1 was the mechanism of drug resistance, in the human cancers studied, which might therefore be a new target for MDR reversal therapy.

\section{Acknowledgments}

This work was supported by grants from the National Foundation of Natural Sciences, China (no. 30971134) and the Ministry of Public Health, China (no. 200802094).

\section{References}

1. Szakács G, Paterson JK, Ludwig JA, Booth-Genthe C and Gottesman MM: Targeting multidrug resistance in cancer. Nat Rev Drug Discov 5: 219-234, 2006.

2. Lage $H$ : An overview of cancer multidrug resistance: a still unsolved problem. Cell Mol Life Sci 65: 3145-3167, 2008. 
3. Shibayama H, Takai E, Matsumura I, et al: Identification of a cytokine-induced antiapoptotic molecule anamorsin essential for definitive hematopoiesis. J Exp Med 199: 581-592, 2004.

4. Hao Z, Li X, Qiao T, Du R, Hong L and Fan D: CIAPIN1 confers multidrug resistance by upregulating the expression of MDR-1 and MRP-1 in gastric cancer cells. Cancer Biol Ther 5: 261-266, 2006

5. Li X, Hong L, Zhao Y, et al: A new apoptosis inhibitor, CIAPIN1 (cytokine-induced apoptosis inhibitor 1), mediates multidrug resistance in leukemia cells by regulating MDR-1, Bcl-2, and Bax. Biochem Cell Biol 85: 741-750, 2007.

6. Giovannetti E, Backus HH, Wouters D and Peters GJ: Functional inactivity and mutations of p53 differentially affect sensitivity to 5-fluorouracil and antifolate inhibitors of thymidylate synthase (TS) by altering TS levels in colorectal cancer cells. Nucleosides Nucleotides Nucleic Acids 27: 740-745, 2008.

7. Kantharidis P, El-Osta A, de Silva M, et al: Altered methylation of the human MDR1 promoter is associated with acquired multidrug resistance. Clin Cancer Res 3: 2025-2032, 1997.

8. Peart MJ, Tainton KM, Ruefli AA, et al: Novel mechanisms of apoptosis induced by histone deacetylase inhibitors. Cancer Res 63: 4460-4471, 2003.

9. Ma Q, Zhang ZS, Zhong SS and Lai ZS: Uptake and distribution of adriamycin in multidrug-resistant LoVo/Adr cells. Di Yi Jun Yi Da Xue Xue Bao 22: 264-266, 2002.

10. Hao Z, Qiao T, Jin X, Li X, Gao J and Fan D: Preparation and characterization of a specific monoclonal antibody against CIAPIN1. Hybridoma (Larchmt) 24: 141-145, 2005.

11. Li X, Pan Y, Fan R, et al: Adenovirus-delivered CIAPIN1 small interfering RNA inhibits HCC growth in vitro and in vivo. Carcinogenesis 29: 1587-1593, 2008.

12. Tchénio T, Havard M, Martinez LA and Dautry F: Heat shockindependent induction of multidrug resistance by heat shock factor 1. Mol Cell Biol 26: 580-591, 2006.

13. Zhang Y, Shi Y, Li X, et al: Proteasome inhibitor MG132 reverses multidrug resistance of gastric cancer through enhancing apoptosis and inhibiting P-gp. Cancer Biol Ther 7: 540-546, 2008.

14. Hong L, Piao Y, Han Y, et al: Zinc ribbon domain-containing 1 (ZNRD1) mediates multidrug resistance of leukemia cells through regulation of P-glycoprotein and Bcl-2. Mol Cancer Ther 4: 1936-1942, 2005.

15. Baumert $\mathrm{C}$ and Hilgeroth $\mathrm{A}$ : Recent advances in the development of P-gp inhibitors. Anticancer Agents Med Chem 9: 415-436, 2009.

16. Han Z, Hong L, Han Y, et al: Phospho Akt mediates multidrug resistance of gastric cancer cells through regulation of $\mathrm{P}$-gp, Bcl-2 and Bax. J Exp Clin Cancer Res 26: 261-268, 2007.
17. Hao Z, Li X, Qiao T, Du R, Zhang G and Fan D: Subcellular localization of CIAPIN1. J Histochem Cytochem 54: 1437-1444, 2006.

18. Li Y, Yuan H, Yang K, Xu W, Tang W and Li X: The structure and functions of P-glycoprotein. Curr Med Chem 17: 786-800, 2010.

19. Gogvadze V, Orrenius S and Zhivotovsky B: Mitochondria as targets for chemotherapy. Apoptosis 14: 624-640, 2009.

20. Cory S and Adams JM: Killing cancer cells by flipping the Bcl-2/Bax switch. Cancer Cell 8: 5-6, 2005.

21. He L, Wang $\mathrm{H}$, Jin $\mathrm{H}$, et al: CIAPIN1 inhibits the growth and proliferation of clear cell renal cell carcinoma. Cancer Lett 276: 88-94, 2009.

22. Yu H and Jove R: The STATs of cancer - new molecular targets come of age. Nat Rev Cancer 4: 97-105, 2004.

23. Ravi R, Jain AJ, Schulick RD, et al: Elimination of hepatic metastases of colon cancer cells via p53-independent cross-talk between irinotecan and Apo2 ligand/TRAIL. Cancer Res 64: 9105-9114, 2004

24. Xiong H Zhang ZG, Tian XQ, et al: Inhibition of JAK1, 2/STAT3 signaling induces apoptosis, cell cycle arrest, and reduces tumor cell invasion in colorectal cancer cells. Neoplasia 10: 287-297, 2008.

25. Yang, LY and Trujillo JM: Biological characterization of multidrug-resistant human colon carcinoma sublines induced/selected by two methods. Cancer Res 50: 3218-3225, 1990.

26. Jiang XH, Wong BC, Lin MC, et al: Functional p53 is required for triptolide-induced apoptosis and AP-1 and nuclear factor-kappaB activation in gastric cancer cells. Oncogene 20: 8009-8018, 2001.

27. El-Mahdy MA, Zhu Q, Wang QE, Wani G and Wani AA Thymoquinone induces apoptosis through activation of caspase- 8 and mitochondrial events in p53-null myeloblastic leukemia HL-60 cells. Int J Cancer 117: 409-417, 2005.

28. Chen YN, Mickley LA, Schwartz AM, Acton EM, Hwang JL and Fojo AT: Characterization of adriamycin-resistant human breast cancer cells which display overexpression of a novel resistancerelated membrane protein. J Biol Chem 265: 10073-10080, 1990.

29. Nita ME, Nagawa H, Tominaga O, et al: 5-Fluorouracil induces apoptosis in human colon cancer cell lines with modulation of Bcl-2 family proteins. Br J Cancer 78: 986-992, 1998.

30. Sax JK and El-Deiry WS: P53 downstream targets and chemosensitivity. Cell Death Differ 10: 413-417, 2003. 